\title{
Movement based time division multiplexing for near real time feedback body area network applications
}

\author{
Haider A. Sabti \& David V. Thiel \\ Centre for Wireless Monitoring and Applications, Griffith University, Brisbane, QLD Australia \\ haider.al-husseinawi@griffithuni.edu.au,d.thiel@griffith.edu.au
}

\begin{abstract}
A time division multiplexed coding system was designed and tested for a body-centric star network using a wireless node mounted in six different places in different time lots on the arm and leg and a central node (hub) on the chest during running. The proposed network allows a coordinated approach to gait analysis. The time sequence for communications between the wireless nodes and the hub is set during the first few steps as runners have different styles and can run at different speeds. After calibration the central unit sends a synchronization pulse during every running step and sets a unique transmission time window for each individual node. The time windows are scheduled when there is reliable communications between the hub and the sensor nodes around the body. Accelerometers on each node are used to identify these time windows for the diverse angles of rotation of the human limbs during running.
\end{abstract}

Keywords-WBAN; WPAN; BAN; Body Centric Network; Body Area Network; Time division; Sports; Running

\section{INTRODUCTION}

The growing adoption of Wireless Body Area Networks (WBAN) has led to improvements in this technology through antenna design and communications strategies. Sensors implanted in or on the human body and clothing [1], (so called smart clothing) are being rapidly adopted in health and sports monitoring applications [2]. The feedback provided by these wireless nodes can help to study patient health and athlete performance in real time $[3,4]$. However, during normal body movements the limbs interfere with the line of sight (LOS) of the transmitting and receiving nodes resulting in multipath fading and scattering effect which makes it very hard to sustain continuous wireless communications [5]. In running, the time between each two foot contacts with the ground of the right and left leg is called the swing time, and during the natural human running movement the swing time is identical for opposite legs and arms [6]. The use of multiple access technologies provide consistent and burst signal transmission over time, frequency and code [7]; which can support the reliable communication for the wireless nodes without packet collision. This paper presents the use of a time division technique to allocate time segments for each on-body wireless node to transmit near real time data during running to the wireless central node for further processing.
The wireless sensor node consisted of a MCU (Microcontroller), transceiver chip, accelerometer sensor and memory. The node have UART interface allows wired data transfers to a local computer for monitoring or further processing during or after the completion of the sport activity. It has a Built-in antenna works in the $2.4-2.5 \mathrm{GHz}$ universal ISM band, and supports up to six channels of data reception. It was programmed to perform it tasks in C language with Atme Studio 6.1 to implement the codes.

\section{METHODOLOGY}

A wireless transceiver node was placed at six different locations on the legs and arms of the human body and the participant was asked to assume stationary positions that are characteristic of running movements [8]. To make sure the wearable wireless node is stable during movement and for easily on body mounting, the nodes were attached in fabric bands to the outside part of the wrist and arm (above the elbow), the upper surface of the foot, the lower and upper parts of the leg and on the thigh just above the knee (shown in Fig. 1). The received signal level was monitored as a function of limb position using a small, portable RF receiver with a sensitivity threshold that allows a transmission data rate of $250 \mathrm{Kbps}$ at $2.45 \mathrm{GHz}$. The measurements were taken in free space to minimize possible multipath effects from nearby objects.

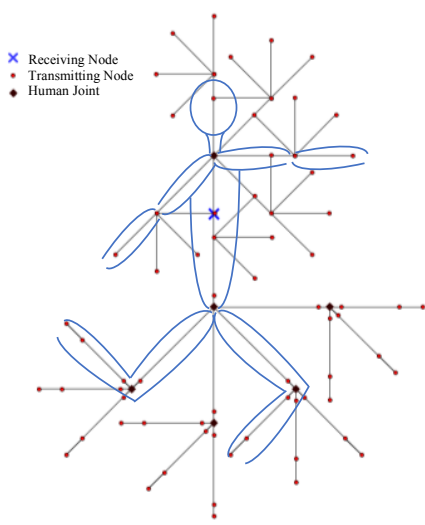

Fig. 1. Human model shows the receiving and transmitting nodes for all leg and arm positions. 


\section{EXPERIMENTAL RESULTS}

This paper reports a real time feedback WBAN using movement based time division multiplexing and follows previous work on analysis of the wireless link performance in body area networks [9].The recorded data for each running position revealed a set of angular windows for each sensor location at which a reliable wireless communication can occur. These angular windows are repetitive in a periodic movement such as running and can be converted into time windows when the swing time of runner has been determined. During the calibration mode of the proposed technique shown in Fig. 2, the time window calculations for different runners is done by comparing the accelerometer data for a number of swings in order to provide the most accurate swing value. In one example, the swing time used to calculate the time windows for each wireless node was set to $400 \mathrm{~ms}$. This value was selected because the variation in the swing time between human arms and legs of different runners is very small at their top speed (around $400 \mathrm{~ms}$ ) [6].

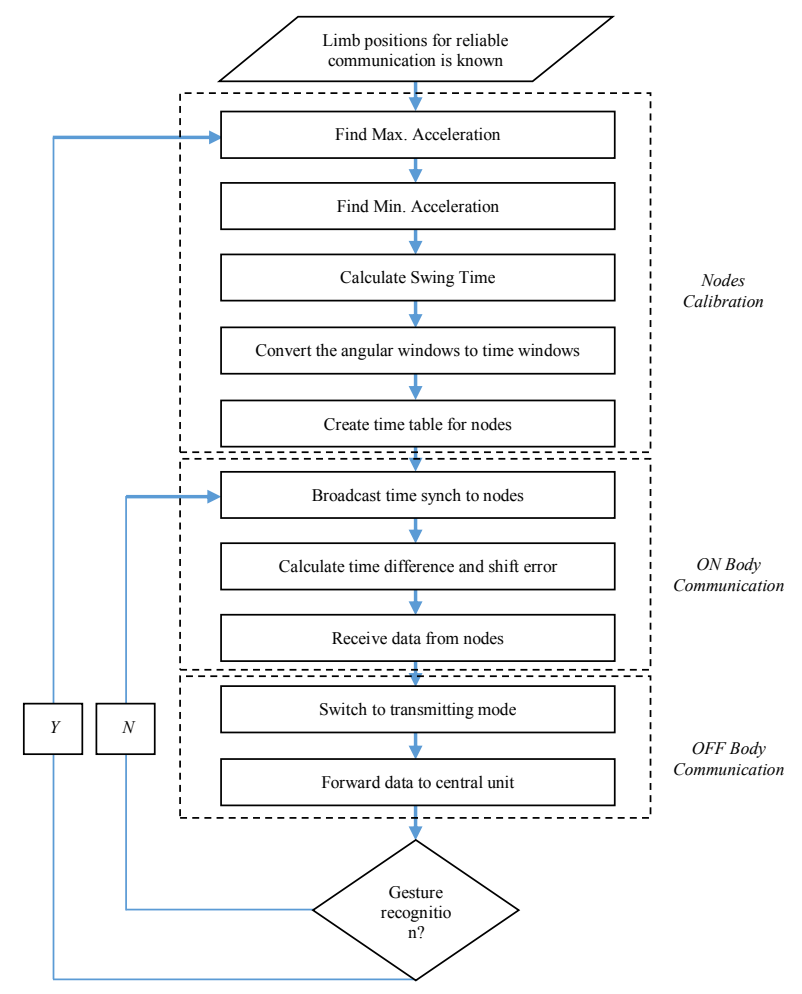

Fig. 2. Flow chart describe the node calibration and (ON body and OFF body) communication process of the hub node.

Table I shows the time windows relative to the swing time during running. The cycle takes two swing times and begins at $0 \mathrm{~ms}$ when one leg and opposing arm stretch far behind the body while reaching the extreme position in front of the body at $400 \mathrm{~ms}$ and ends back at $800 \mathrm{~ms}$ (Fig. 3).
Table I. Sensor time windows relative to the swing time in running.

\begin{tabular}{|c|c|c|c|c|c|c|}
\hline \multirow{2}{*}{ Time (ms) } & \multicolumn{7}{|c|}{ Sensor mounting location } \\
\cline { 2 - 7 } & Wrist & Foot & Lower Leg & Upper Leg & Thigh & Arm \\
\hline Start time & 121 & 223 & 277 & 245 & 245 & 382 \\
\hline End Time & 400 & 400 & 389 & 353 & 335 & 384 \\
\hline Total Time & 279 & 177 & 163 & 108 & 90 & 2 \\
\hline
\end{tabular}

In Table I, the longest time windows for continuous reliable communication is provided for a wireless node attached to the wrist or foot of the human body. The provision of LOS between the chest and wrist/foot during the course of the second swing allows the nodes to preserve consistent wireless connectivity. Converting these time windows to data values determines the connect time and so the transmission capacity of each sensor location in a swing period. It shows that for a maximum data rate of $250 \mathrm{~Kb} / \mathrm{s}$, a total traffic of nearly $8.75 \mathrm{~KB}$ can be provided when the sensor is attached to the wrist during a single swing time while only 55.6 bytes for arm positions.

Gait analysis requires the capture of body movement actions such as walking or running. The most common methods use video recording and subsequent image processing using complicated analysis algorithms [10]. The new concept for capturing the human movement is given by using three axis accelerometer sensors with 100 samples/s per channel. Each sensor records 80 samples per two swing actions and sends these samples at the allocated time window to the central unit which in turn will sends them off the body (locally or to the cloud) for further analysis. The data sent from each sensor (excluding the overhead) is 480 bytes because each single 3 axis accelerometer sample equals 6 bytes. With a data rate of $250 \mathrm{Kbps}$ each sensor will require a time burst duration of $15.36 \mathrm{~ms}$ to transmit the sampled data to the central node. This equates to five times the period available to retransmit the collected data. The hub node send synchronization pulses each $400 \mathrm{~ms}$, however the nodes receive these pulses each $800 \mathrm{~ms}$ due to the swinging action of right and left limbs during running. As a result, the nodes have to maintain time for 800 ms depending on their local clock and this might produce a time shift from the hub node time which can be corrected using a simple version of two nodes time shift error correction algorithm [11].

Fig. 3 shows the time table for synchronization, data collection and transmission. As the running is a periodic movement only three swing actions are presented. After calibration, it is not significant whether the right or left leg/arm will give the first swing. In this example the first swing start at $\mathrm{t}=0$ and was assumed to occur when the right leg and left arm move forward and the second swing is when they reverse position. At the first swing, the central node broadcasts its time and a time table of transmission for each node so that each wireless node is synchronized with the central node. After that, the node switches to receive mode to collect the data from the wireless sensors attached to the left wrist and right leg. 


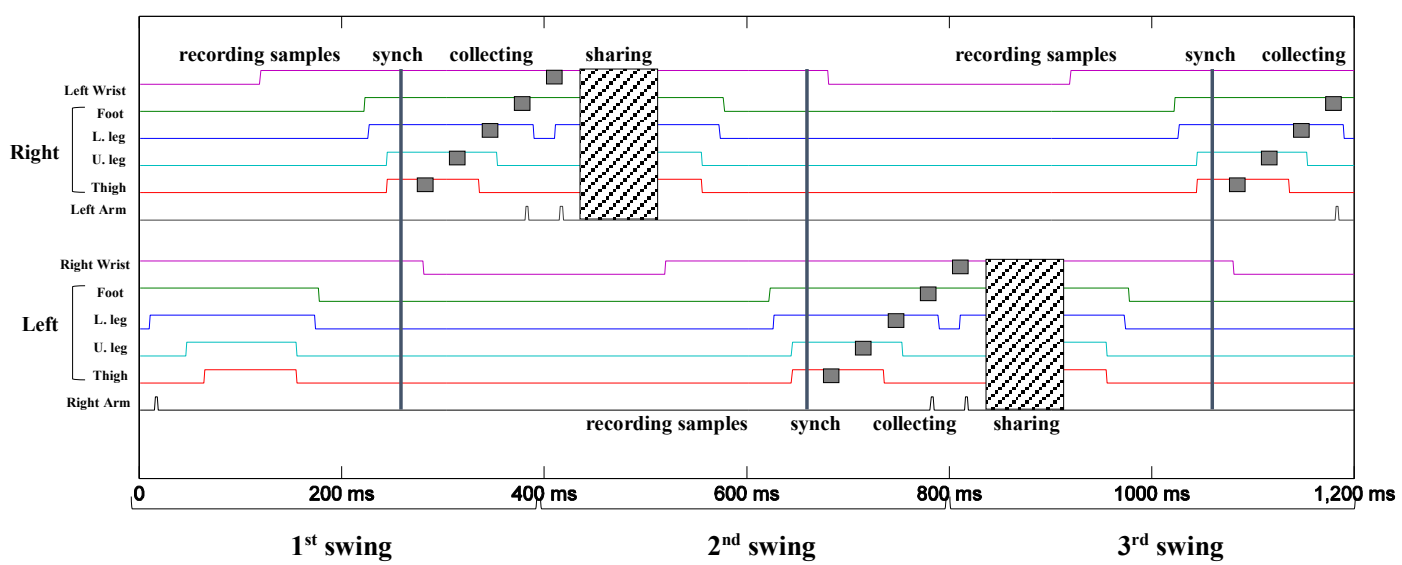

Fig. 3. Wireless nodes time allocation and synchronization during running.

The process of synchronization and data collection takes nearly $170 \mathrm{~ms}$ after which the central wireless node forwards this information to a local computer to provide a near real time feedback data. The delay between the sensor signals and computer recorded data is less than one second. The same procedure is repeated at the second swing, however this time the data sets collected and transmitted are for the right wrist and left leg and the process continues along the running action.

\section{CONCLUSION}

A new method for capturing gait movement is presented and explained. The method uses a time division technique and swing time of runners to set the transmission time windows for the sensors distributed on the human body. The accelerometer on each sensor node helps identify these time windows during the action of running. The synchronization process is carried out by the central node to ensure that the data collected were sampled at the same time for each wireless node. Future work will be based on the dynamic measurements of the algorithm for a variety of different runners to study the influence of running experience, running style and body measurements. It is anticipated that this study can give useful information to athletics coaches and the runners themselves, to improve running technique.

\section{REFERENCES}

[1] B. Latré, B. Braem, I. Moerman, C. Blondia, and P. Demeester, "A survey on wireless body area networks," Wirel. Netw., vol. 17, pp. 1-18, 2011.

[2] L. M. Borges, A. Rente, F. J. Velez, L. R. Salvado, A. S. Lebres, J. M. Oliveira, et al., "Overview of progress in Smart-Clothing project for health monitoring and sport applications," in Applied Sciences on Biomedical and Communication Technologies, 2008. ISABEL '08. First International Symposium on, 2008, pp. 1-6.

[3] D. Brunelli, E. Farella, L. Rocchi, M. Dozza, L. Chiari, and L. Benini, "Bio-feedback system for rehabilitation based on a wireless body area network," in Pervasive Computing and Communications Workshops, 2006. PerCom Workshops 2006. Fourth Annual IEEE International Conference on, 2006, pp. 5 pp.-531.
[4] J. Neville, A. Wixted, D. Rowlands, and D. James, "Accelerometers: An underutilized resource in sports monitoring," in Intelligent Sensors, Sensor Networks and Information Processing (ISSNIP), 2010 Sixth International Conference on, 2010, pp. 287-290.

[5] S. L. Cotton and W. G. Scanlon, "A Statistical Analysis of Indoor Multipath Fading for a Narrowband Wireless Body Area Network," in Personal, Indoor and Mobile Radio Communications, 2006 IEEE 17th International Symposium on, 2006, pp. 1-5.

[6] P. G. Weyand, D. B. Sternlight, M. J. Bellizzi, and S. Wright, "Faster top running speeds are achieved with greater ground forces not more rapid leg movements," Journal of Applied Physiology, November 1, 2000, vol. 89, pp. 1991-1999.

[7] C. Hsiao-Hwa and M. Guizani, "Multiple access technologies for B3G wireless communications," Communications Magazine, IEEE, 2005, vol. 43, pp. 65-67.

[8] C. L. Vaughan, B. L. Davis, and J. C. O. Connor, DYNAMICS OF HUMAN GAIT, Second Edition ed. Cape Town, South Africa: Kiboho Publishers, 1999.

[9] H. A. Sabti and D. V. Thiel, "A study of wireless communication links on body centric network during running," submitted to the International Sports Engineering Association, Sheffield UK, 2014. [under review]

[10] S. Sarkar, P. J. Phillips, Z. Liu, I. R. Vega, P. Grother, and K. W. Bowyer, "The humanID gait challenge problem: data sets, performance, and analysis," IEEE Trans Pattern Anal Mach Intell, Feb 2005, vol. 27, pp. 162-77.

[11] M. K. Maggs, S. G. O'Keefe, and D. V. Thiel, "Consensus Clock Synchronization for Wireless Sensor Networks," IEEE Sensors Journal, Jun 2012, vol. 12, pp. 2269-2277. 